\title{
Frecuencia y características demográficas de pacientes con diagnóstico de trombosis de stents coronarios implantados en un hospital universitario entre enero de 2011 a diciembre de 2012
}

\author{
Bonell Patiño*, Sonia Hernández, Juan S. Segura, Julio C. Rojas, \\ Manuel A. Ramírez, Húber Sánchez y Jorge Villegas
}

Departamento de Medicina Interna y Cardiología-Hemodinámica, Hospital Universitario Clínica San Rafael, Bogotá, Colombia

Recibido el 25 de febrero de 2015; aceptado el 25 de mayo de 2015

Disponible en Internet el 29 de agosto de 2015

\author{
PALABRAS CLAVE \\ Stent; \\ Trombosis; \\ Intervención \\ coronaria percutánea
}

\begin{abstract}
Resumen
Objetivo: Establecer la frecuencia y las características demográficas de pacientes con diagnóstico de trombosis de stent coronario atendidos en el Hospital Universitario Clínica San Rafael, de Bogotá, durante un periodo de 24 meses.

Métodos: Estudio descriptivo retrospectivo en el que se revisaron las historias clínicas de pacientes mayores de 18 años con diagnóstico de trombosis de stent coronario confirmado mediante arteriografías realizadas en dicha institución en un lapso de 24 meses, atendidos de forma ambulatoria, en los departamentos de urgencias y/o hospitalización.

Resultados: Se realizaron 890 arteriografías con angioplastia e implante de stent, en las que se encontró un $2,7 \%$ de frecuencia de trombosis del stent; en el $70 \%$ los hombres presentaron un promedio de edad menor respecto a las mujeres (63,0 vs. 70,3 años). Las comorbilidades más frecuentes fueron: hipertensión arterial en el 65\%, diabetes mellitus en el $25 \%$ y enfermedad renal crónica en el $10 \%$ de los casos. El promedio de fracción de eyección del ventrículo izquierdo fue del $38,7 \%$. Incidentalmente, durante el análisis de datos se encontró fenómeno de reestenosis del stent en el $14,5 \%(n=36)$ entre quienes se les hizo angioplastia más implante de stent.

Conclusiones: La frecuencia de trombosis del stent coronario en la población analizada es similar a la reportada en la literatura mundial. Es más común en el sexo masculino, a una edad de aparición más temprana asociada con un deterioro marcado de la función ventricular izquierda. La alta frecuencia de evento de reestenosis del stent requiere más estudios en el futuro.

(c) 2015 Sociedad Colombiana de Cardiología y Cirugía Cardiovascular. Publicado por Elsevier España, S.L.U. Este es un artículo Open Access bajo la licencia CC BY-NC-ND (http://creativecommons.org/licenses/by-nc-nd/4.0/).
\end{abstract}

\footnotetext{
* Autor para correspondencia.

Correo electrónico: bonell32@hotmail.com (B. Patiño).
}

http://dx.doi.org/10.1016/j.rccar.2015.05.010

0120-5633/@ 2015 Sociedad Colombiana de Cardiología y Cirugía Cardiovascular. Publicado por Elsevier España, S.L.U. Este es un artículo Open Access bajo la licencia CC BY-NC-ND (http://creativecommons.org/licenses/by-nc-nd/4.0/). 


\section{KEYWORDS}

Stent;

Thrombosis;

Percutaneous

coronary intervention
Frequency and demographic characteristics of patients diagnosed thrombosis coronary stent implantation in a university hospital from January 2011 to December 2012

\begin{abstract}
Objective: Establish frequency and demographic characteristics of patients with diagnosis of coronary stent thrombosis into Hospital Universitario Clínica San Rafael (HUCSR) over a time period of 24 months.

Methods: Retrospective and descriptive Study. Clinical records were reviewed for patients older 18 with diagnosis of coronary stent thrombosis, confirmed through coronary arteriography made in HUCSR-Hemodinamics department on urgency, inpatient or outpatient, on a 24-month time period.

Results: 890 arteriography and angioplasty plus stent implantation were performed. Frequency of coronary stent thrombosis was of $2.7 \%$, in $70 \%$ of the cases male patients were younger on average than women (63.0 years old vs 70.3 years old). Most commonly described associated diseases were arterial hypertension (65\%), diabetes mellitus (25\%), and chronic kidney disease $(10 \%)$. The average left ventricular ejection fraction was $38.7 \%$. Incidentally, while data were being recollected, the stent stenosis was found in too many patients, with a frequency of $14.5 \%$ $(n=36)$ on patients who underwent angioplasty and stent implantation.

Conclusions: Coronary stent thrombosis frequency is similar to reports around the world. This complication is more frequent in male patients, onset age was younger and associated with worse left ventricular function. High coronary stent stenosis frequency required more studies. (c) 2015 Sociedad Colombiana de Cardiología y Cirugía Cardiovascular. Published by Elsevier España, S.L.U. This is an open access article under the CC BY-NC-ND license (http://creativecommons.org/licenses/by-nc-nd/4.0/).
\end{abstract}

\section{Introducción}

Durante los últimos años la intervención coronaria percutánea, o angiografía coronaria, es un procedimiento con el cual se logra establecer el sitio de obstrucción y dar un tratamiento dirigido. Esta técnica ha alcanzado mejoras progresivas en el éxito, la seguridad y la durabilidad de sus resultados, pero así como cada innovación resuelve problemas anteriores, también suele introducir nuevos eventos adversos, tal como ha sucedido con la reestenosis o trombosis intrastent ${ }^{1,2}$.

En este sentido, el stent liberador de drogas inmunosupresoras (drug eluting stent [DES]) es el avance más reciente de la terapia intervencionista para el tratamiento de las oclusiones arteriales coronarias ${ }^{3,4}$.

De otro lado, en cuanto a la enfermedad coronaria, desde la introducción del stent se generó un impacto positivo en la reducción de las complicaciones y la mortalidad en la enfermedad, pero también se conoció que su potencial riesgo es la trombosis tardía del stent, que se posiciona como la problemática más frecuente de la implantación de estos dispositivos, cuya solución es la terapia antiagregante doble con ácido acetilsalicílico (AAS) y clopidogrel con posterior continuidad de pequeñas dosis de AAS en forma indefinida. Siguiendo esta pauta, luego de 30 días del implante ocurre trombosis del stent en menos del $1 \%$ de los pacientes ${ }^{3,4}$.

Existen factores clínicos que predisponen, como la edad, la diabetes mellitus, la fracción de eyección baja o el incumplimiento terapéutico antiagregante; otros factores son los angiográficos, como las lesiones largas, complejas, con trombos o lechos vasculares enfermos.
Finalmente existen factores directamente relacionados con la intervención y que son relativos a la implantación del stent y al resultado angiográfico obtenido. Como es apreciable, muchos de estos factores no son modificables si se exceptúan los concernientes a la implantación del stent 0 al tratamiento antiagregante combinado preventivo $0^{4,5}$.

En Colombia, de acuerdo con estadísticas publicadas por el Ministerio de la Protección Social en el informe sobre la Situación de salud en Colombia-Indicadores de salud 2007, la enfermedad isquémica del corazón es la principal causa de muerte tanto en hombres como en mujeres mayores de 45 años, e incluso supera las muertes violentas o los cánceres combinados. La tasa de mortalidad atribuible a esta enfermedad fue de 107,3 por 100.000 habitantes en personas de 45 a 64 años, y de 867,1 por 100.000 habitantes para personas de 65 años o más. Sin embargo, en el país se desconoce globalmente la magnitud actual de la carga de la enfermedad cardíaca isquémica en términos de prevalencia de factores de riesgo, a excepción de la obesidad y el sedentarismo; por tanto, se necesitan estudios epidemiológicos globales sobre este aspecto ${ }^{6}$.

Los stents liberadores de fármaco requieren un período más amplio de terapia antiplaquetaria combinada hasta que la endotelización se haya completado; infortunadamente, no se sabe cuándo ocurrirá ${ }^{3}$. Por lo tanto, se recomienda la administración de clopidogrel mínimo hasta tresmeses después de la colocación del stent, en el caso de liberar sirolimus, y hasta seismeses, en caso de paclitaxel. Recientemente han aparecido guías terapéuticas en las que se recomienda el mantenimiento de clopidogrel al menos durante un año. Sea cual sea la pauta elegida, la 
interrupción anticipada de la terapia antiplaquetaria es el factor más importante para la trombosis del stent.

En los primeros estudios que evaluaron la incidencia de trombosis del stent, tras la generalización de la terapia antiagregante combinada se identificó su prevalencia dentro de los primeros 30 días, siendo independiente el tipo de stent a utilizar; los principales predictores de trombosis identificados fueron la existencia de disección residual, la presencia de trombo en la lesión tratada y la falta de adherencia o resistencia al manejo farmacológico.

Existen múltiples estudios; el de Berna-Rotterdam, con 8.146 pacientes, presenta una incidencia del $2,9 \%$ de reestenosis temprana (aguda y subaguda) y del 0,6\% tardía a un año. Dentro de la literatura está el registro alemán-italiano y el sueco (SCAAR), con incidencias del 1,2\% de trombosis temprana ${ }^{3,7}$.

Sin embargo, en Colombia no hay hasta la fecha estudios que definan claramente las posibles variables de las complicaciones asociadas a la trombosis del stent. Ante esta perspectiva se desarrolló un trabajo retrospectivo basado en la revisión de historias clínicas de personas mayores de 18 años que fueron llevadas a arteriografía coronaria entre enero de 2011 a diciembre de 2012 (quienes consultaron por urgencias, servicios ambulatorios u hospitalización), con el fin de establecer la frecuencia y las características demográficas de pacientes con diagnóstico de trombosis de stent coronario atendidas en el servicio de hemodinamia de un hospital universitario.

\section{Métodos}

\section{Tipo de estudio}

Estudio descriptivo retrospectivo, dividido en dos fases clínicas: fase I, realizada con datos desde el 1 de enero de 2011 hasta el 30 de noviembre de 2011, y fase I, llevada a cabo desde el 1 de diciembre de 2011 hasta el 31 de diciembre de 2012 (24 meses).

\section{Población}

Pacientes mayores de 18 años con diagnóstico de trombosis del stent coronario confirmado por cateterismo cardiaco realizado en el Hospital Universitario Clínica San Rafael durante el periodo en mención.

\section{Variables y nivel de medición}

\section{Variables nominales}

Sexo, tipo de stent implantado, suspensión precoz de antiagregantes, disfunción sistólica del ventrículo izquierdo, enfermedad coronaria, hipertensión arterial, enfermedad renal crónica, diabetes mellitus, localización del stent, ámbito de atención: urgencias, ambulatorio, hospitalización.

\section{Variables ordinales}

$\mathrm{TIMI}<3$ (flujo distal rápido) demostrado mediante angiografía.
Variable de razón

Edad, número de stents, fracción de eyección del ventrículo izquierdo, número de pacientes.

\section{Recolección de datos}

Se realizó una revisión en el sistema de Historia Clínica Virtual «HeOn», del Hospital Universitario Clínica San Rafael, de los pacientes atendidos de forma ambulatoria o por urgencias y/o hospitalización en el departamento de hemodinamia, a quienes se les realizó arteriografía coronaria, en el periodo comprendido entre el 1 de enero de 2011 al 31 de diciembre de 2012, previa autorización del comité de ética de la institución.

Una vez identificados los pacientes con diagnóstico de trombosis de stent coronario, se elaboró una tabla operativa de datos en donde se consignaron las variables.

\section{Tamaño de la muestra}

Se realizó cálculo del tamaño de la muestra en un escenario de proporción esperada del $5 \%$, con un nivel de confianza del $95 \%$, tamaño de la muestra determinada en 73 pacientes como población total.

\section{Plan de análisis}

Los datos obtenidos se organizaron en distribuciones de frecuencia que se presentaron en tablas operativas y gráficas; las variables numéricas se calcularon con medidas de tendencia central y de dispersión, y los principales resultados se midieron como frecuencia. Se buscó establecer diferencias estadísticas entre las variables y sus características de interés por EpiOpen v.3, el análisis estadístico en tablas de $2 \times 2$ para obtener resultados mediante Chi cuadrado $\left(\chi^{2}\right)$, la razón de disparidad y sus respectivos intervalos de confianza del $95 \%$.

\section{Resultados}

\section{Fase I}

En el Hospital Universitario Clínica San Rafael se realizaron durante la fase I del estudio correspondiente a datos entre el 1 de enero y el 30 de noviembre de 2011, un total de 643 angioplastias con colocación de stent en pacientes con enfermedad coronaria. El $62,3 \%$ de estas angioplastias se hizo en pacientes de género masculino $(n=400)$ y el $37,7 \%$ en pacientes de género femenino $(n=242)$. El promedio de edad en dicha población fue de 65,1 años. La mayor parte de los procedimientos se llevó a cabo en pacientes hospitalizados (59,3\%), mientras que el $35,8 \%$ se efectuó de manera ambulatoria; esta información no se encontró registrada en 28 casos $(4,4 \%)$. Del total de la población, 10 pacientes presentaron trombosis del stent, correspondiente a una frecuencia del 1,6\%. El $60 \%$ de los pacientes correspondió al género masculino, con un promedio de edad de 67,5 años. El 2,7\% estaba hospitalizado (tabla 1).

En los pacientes no trombosados se encontró con mayor frecuencia hipertensión arterial y en menor proporción 
Tabla 1 Tipo de ingreso de los pacientes llevados a arteriografía coronaria durante la fase ı en el Hospital Universitario Clínica San Rafael

\begin{tabular}{lll}
\hline Ámbito de atención & $\begin{array}{l}\text { Stents no } \\
\text { trombosados }\end{array}$ & $\begin{array}{l}\text { Stents } \\
\text { trombosados }\end{array}$ \\
\hline Hospitalizado $(59,3 \%)$ & 375 & 10 \\
Ambulatorio $(35,8 \%)$ & 229 & 0 \\
Total & 633 & 10 \\
\hline
\end{tabular}

Tabla 2 Comorbilidades entre pacientes llevados a arteriografía coronaria en el Hospital Universitario Clínica San Rafael durante la fase I

\begin{tabular}{lll}
\hline Patología & No trombosados & Trombosados \\
\hline Hipertensión arterial & $373(59 \%)$ & $6(60 \%)$ \\
Diabetes mellitus & $152(24,1 \%)$ & $2(20 \%)$ \\
Enfermedad coronaria & $270(42,7 \%)$ & $7(70 \%)$ \\
Enfermedad renal crónica & $68(10,8 \%)$ & $1(10 \%)$ \\
\hline
\end{tabular}

enfermedad renal. La patología asociada más común en los pacientes que presentaron trombosis fue la enfermedad coronaria, seguida de la hipertensión arterial (tabla 2), y la totalidad de estos estaba hospitalizada al momento de la complicación.

En la población analizada se encontró una fracción de eyección entre el 10 y el 70\%, con un promedio del 45,29\%; es así como en pacientes que presentaron la complicación de trombosis del stent se encontró una fracción de eyección del $36 \%$, con un valor mínimo del $15 \%$ y máximo del $63 \%$ (tabla 3).

En 2 pacientes se documentó que la causa probable de la trombosis fue el no consumo de clopidogrel; en 4 casos se utilizaron stents metálicos y en 6 , stents medicados. El vaso coronario más comprometido fue la descendente anterior, y en general tuvo una prevalencia mayor en pacientes del sexo masculino (tabla 3 ).
Tabla 4 Total de procedimientos en hemodinamia, fase ॥

\begin{tabular}{ll}
\hline Coronarios & 1.230 \\
No coronarios & 228 \\
Total & 1.458 \\
\hline
\end{tabular}

Tabla 5 Angioplastia + implante de stent, fase ॥

\begin{tabular}{llll}
\hline Sexo & Número & Promedio edad & Porcentaje \\
\hline Hombres & 185 & 64,5 & $74,50 \%$ \\
Mujeres & 63 & 69,8 & $25,40 \%$ \\
Total & 248 & 67,15 & $100 \%$ \\
\hline
\end{tabular}

\section{Fase II}

En el hospital Universitario Clínica San Rafael se realizaron en el periodo correspondiente a la segunda fase, entre el 1 de diciembre de 2011 al 31 de diciembre de 2012, un total de 1.458 procedimientos en el servicio de hemodinamia, de los cuales 1.230 correspondieron a diagnóstico y tratamiento de enfermedad coronaria y 228 no relacionados con enfermedad coronaria (tabla 4).

De 1.230 procedimientos concernientes a enfermedad coronaria, se realizaron 248 angioplastias más implante de stent en pacientes con enfermedad coronaria. El $74,5 \%$ de las angioplastias se realizaron en pacientes de sexo masculino $(n=185)$ y el $25,5 \%$ en pacientes de sexo femenino $(n=63)$. El promedio de edad fue de 67,1 años, con menor y mayor edad (tabla 5).

Del total de procedimientos endovasculares de angioplastia más implante de stent, se encontraron 10 pacientes con trombosis del stent, que corresponden a una frecuencia del $4 \%$.

De 10 pacientes con diagnóstico angiográfico de trombosis del stent, la totalidad se reportó en el ámbito hospitalario en el contexto de urgencias. De estos pacientes, un $80 \%$ correspondió al sexo masculino $(n=8)$ y $20 \%$ al femenino $(n=2)$, con un promedio de edad de 62,8 años para el primer

Tabla 3 Características demográficas de pacientes con diagnóstico de trombosis del stent, fase I

\begin{tabular}{|c|c|c|c|c|c|c|c|c|c|c|c|}
\hline Paciente & Sexo & Edad & HTA & Enf. cor. & $\mathrm{DM} 2$ & ERC & FEVI & DES & BMS & Localización & Susp. Fco \\
\hline 1 & $M$ & 78 & & $x$ & & & $35 \%$ & & $x$ & DA & Sí \\
\hline 2 & $M$ & 65 & & $x$ & & & - & $x$ & & $C D$ & Sí \\
\hline 3 & M & 65 & & $x$ & & & - & $x$ & & DA & No \\
\hline 4 & $M$ & 65 & $x$ & $x$ & & & $45 \%$ & & $x$ & $C D$ & No \\
\hline 5 & $M$ & 56 & & $x$ & & & $63 \%$ & $x$ & & DA & No \\
\hline 6 & $M$ & 51 & $x$ & $x$ & & & $25 \%$ & $x$ & & $C X$ & No \\
\hline 7 & $\mathrm{~F}$ & 67 & $x$ & $x$ & & & - & $x$ & & DA & No \\
\hline 8 & $\mathrm{~F}$ & 82 & $x$ & $x$ & $x$ & $x$ & $15 \%$ & $x$ & & DA & No \\
\hline 9 & $\mathrm{~F}$ & 83 & $x$ & $x$ & $x$ & & $15 \%$ & & $x$ & $C D$ & No \\
\hline 10 & $\mathrm{~F}$ & 63 & $x$ & $x$ & & & $60 \%$ & & $x$ & $C X$ & No \\
\hline
\end{tabular}

BMS: bare metal stent, stent metálico o stent convencional; CD: arteria coronaria derecha; CX: arteria circunfleja; DA: arteria descendente anterior; DES: drug eluting stent, stent liberador de droga o stent medicado; DM2: diabetes mellitus tipo 2; DP: arteria descendente posterior; Enf. cor.: enfermedad coronaria; ERC: enfermedad renal crónica; F: femenino; FEVI: fracción de eyección del ventrículo izquierdo; HTA: hipertensión arterial; M: masculino; PL: arteria posterolateral; Susp. fco: suspensión de fármaco, indicando la relación principalmente con la suspensión de clopidogrel.

La edad está reportada en años. 
Tabla 6 Características demográficas de pacientes con diagnóstico de trombosis del stent, fase ॥

\begin{tabular}{llllllllllll}
\hline Paciente & Sexo & Edad & HTA & Enf cor. & DM2 & ERC & FEVI & DES & BMS & Localización & Susp. fco \\
\hline 1 & F & 69 & X & X & & & $25 \%$ & - & X & PL-DP & Sí \\
2 & M & 64 & X & X & X & & $40 \%$ & - & X & CX & No \\
3 & M & 59 & & X & & & $35 \%$ & X & - & CD-CX & No \\
4 & M & 87 & X & X & X & X & $35 \%$ & - & X & CD & No \\
5 & F & 65 & X & X & & & $55 \%$ & X & - & CD & No \\
6 & M & 38 & X & X & & & $50 \%$ & - & X & DP & No \\
7 & M & 50 & & X & & & $30 \%$ & X & - & DA & No \\
8 & M & 68 & & X & & & $50 \%$ & - & X & CD & No \\
9 & M & 66 & X & X & & & $47 \%$ & X & - & DA & No \\
10 & M & 71 & X & X & X & & - & X & - & DA & No \\
\hline
\end{tabular}

BMS: bare metal stent, stent metálico o stent convencional; CD: arteria coronaria derecha; CX: arteria circunfleja; DA: arteria descendente anterior; DES: drug eluting stent, stent liberador de droga o stent medicado; DM2: diabetes mellitus tipo 2; DP: arteria descendente posterior; Enf. cor.: enfermedad coronaria; ERC: enfermedad renal crónica; F: femenino; FEVI: fracción de eyección del ventrículo izquierdo; HTA: hipertensión arterial; M: masculino; PL: arteria posterolateral; Susp. fco: suspensión de fármaco, indicando la relación principalmente con la suspensión de clopidogrel.

La edad está reportada en años.

grupo y de 67,0 años para el segundo, con promedio de edad global de 63,7 años, con edad mínima de 38 y máxima de 87 años.

Las comorbilidades asociadas al grupo de pacientes con trombosis del stent fueron, en orden de frecuencia: enfermedad coronaria $(100 \% ; n=10)$, hipertensión arterial $(70 \%$; $n=7)$, diabetes mellitus $(30 \% ; n=3)$ y enfermedad renal crónica $(10 \% ; n=1)$ (tabla 6$)$.

Dentro del grupo de pacientes con diagnóstico de trombosis del stent, el promedio de fracción de eyección fue del $40,7 \%$, con un valor mínimo del $25 \%$ y máximo del $55 \%$; en uno de los pacientes no se halló este dato. En lo relativo al tipo de stent comprometido, en 2 pacientes se encontró trombosis de 2 stents en cada uno, para un total de 12 stents trombosados, de los cuales 6 (50\%) eran convencionales (BMS: Bare Metal Stent) y $6(50 \%)$ medicados (DES: Drug Eluting Stent) (tabla 6).

Durante la revisión de las historias clínicas se documentó la suspensión del clopidogrel en un solo paciente como causa probable de trombosis del stent. En cuanto a la localización del stent, el vaso más comprometido fue la arteria coronaria derecha en el $33,3 \%$ de los casos $(n=4)$, seguido por la descendente anterior en el $25 \%(n=3)$. Luego se ubican, en orden de mayor a menor frecuencia: la arteria circunfleja y la descendente posterior, con un $16,6 \%$ para cada una $(n=2)$, y la posterolateral, con un $8,3 \%(n=1)$.

Durante el análisis de las historias clínicas de los pacientes que fueron llevados a procedimientos de arteriografía coronaria en la fase II se encontró incidentalmente el fenómeno de reestenosis en varios de los casos, con una frecuencia del 14,5\% ( $n=36)$ dentro del grupo de los que fueron sometidos a angioplastia más implante de stent.

De 36 pacientes con diagnóstico angiográfico de estenosis del stent, el $69,4 \%(n=25)$ se determinó mediante procedimientos realizados de urgencia, mientras el 30,6\% $(n=11)$ fueron de forma programada o ambulatoria (tabla 7).

De este grupo de pacientes, el 33,3\% $(n=12)$ correspondió al género femenino, con un promedio de edad de 70,2 años, con edad mínima de 59 años y máxima de 88 años. Por otra parte, el $66,6 \%(n=24)$ eran del sexo masculino,
Tabla 7 Comparación entre tipo de ingreso y complicación del stent

\begin{tabular}{lll}
\hline Tipo de ingreso & Trombosados & Estenosados \\
\hline Hospitalario & 10 & 25 \\
Ambulatorio & 0 & 11 \\
Frecuencia & $4 \%$ & $14,5 \%$ \\
\hline
\end{tabular}

con un promedio de edad de 62 años, con una edad mínima de 47 años y máxima de 76 años, para un promedio de edad global de 64,7 años.

Las comorbilidades asociadas al grupo de pacientes con estenosis del stent, en orden de frecuencia, fueron: enfermedad coronaria $(100 \% ; n=36)$, hipertensión arterial $(97,2 \% ; n=35)$, diabetes mellitus $(52,7 \% ; n=19)$ y enfermedad renal crónica $(30,5 \% ; n=11)$ (tabla 8$)$.

La fracción de eyección del ventrículo izquierdo promedio fue del $44 \%$, con valor mínimo del $15 \%$ y máximo del $60 \%$; en 6 pacientes no se halló este dato. Se describió un paciente con estenosis de 2 stents y uno con estenosis de 3 stents, para un total de 39 stents con la complicación descrita. En cuanto al tipo de stent, en 8 pacientes no se documentó este dato.

De 28 pacientes en quienes se documentó el tipo de stent implantado (31 stents), el 64,5\% correspondía a stent medicado $(n=20)$ y el $35,5 \%$ a convencional $(n=11)$.

La siguiente fue la localización más común con la complicación mencionada en su orden de frecuencia: arteria

Tabla 8 Comparación de comorbilidades entre pacientes con complicación del stent

\begin{tabular}{|c|c|c|c|c|}
\hline Comorbilidad & Trombosis & $\%$ & Estenosis & $\%$ \\
\hline Hipertensión arterial & 7 & 70 & 35 & 97,20 \\
\hline Diabetes & 3 & 30 & 19 & 52,70 \\
\hline Enfermedad renal crónica & 1 & 10 & 11 & 30,50 \\
\hline Enfermedad coronaria & 10 & 100 & 36 & 100 \\
\hline
\end{tabular}


Tabla 9 Características demográficas de los pacientes con diagnóstico de estenosis del stent

\begin{tabular}{|c|c|c|c|c|c|c|c|c|c|c|c|}
\hline Paciente & Sexo & Edad & Tipo de ingreso & HTA & Enf. cor. & DM2 & ERC & FEVI & DES & BMT & Localización \\
\hline 1 & M & 73 & Hospitalario & $x$ & $x$ & $x$ & $x$ & $45 \%$ & $x$ & NA & DA \\
\hline 2 & $M$ & 63 & Hospitalario & $x$ & $x$ & $x$ & $x$ & $60 \%$ & $x$ & NA & DA \\
\hline 3 & $M$ & 69 & Hospitalario & $x$ & $x$ & - & - & $60 \%$ & NA & $x$ & $C D$ \\
\hline 4 & $M$ & 63 & Hospitalario & $x$ & $x$ & - & $x$ & $15 \%$ & NA & $x$ & $D A-C D$ \\
\hline 5 & $\mathrm{~F}$ & 65 & Hospitalario & $x$ & $x$ & - & - & $60 \%$ & $x$ & NA & $C D$ \\
\hline 6 & M & 66 & Hospitalario & $x$ & $x$ & $x$ & - & $40 \%$ & $x$ & NA & DA \\
\hline 7 & $\mathrm{~F}$ & 88 & Hospitalario & $x$ & $x$ & $x$ & $x$ & $50 \%$ & - & - & DA \\
\hline 8 & M & 62 & Ambulatorio & $x$ & $x$ & - & $x$ & - & - & - & DA \\
\hline 9 & $\mathrm{~F}$ & 75 & Hospitalario & $x$ & $x$ & - & - & $60 \%$ & $x$ & NA & $C D$ \\
\hline 10 & M & 61 & Hospitalario & $x$ & $x$ & - & - & $15 \%$ & $x$ & NA & DA \\
\hline 11 & M & 63 & Hospitalario & $x$ & $x$ & $x$ & $x$ & $20 \%$ & - & - & DA \\
\hline 12 & $M$ & 76 & Hospitalario & $x$ & $x$ & - & - & - & NA & $x$ & $C D$ \\
\hline 13 & M & 65 & Hospitalario & $x$ & $x$ & $x$ & - & $60 \%$ & $x$ & NA & DA \\
\hline 14 & $\mathrm{~F}$ & 59 & Hospitalario & $x$ & $x$ & $x$ & - & $60 \%$ & $x$ & NA & DA \\
\hline 15 & $M$ & 63 & Hospitalario & $x$ & $x$ & - & - & $25 \%$ & NA & $x$ & $\mathrm{RI}$ \\
\hline 16 & M & 73 & Hospitalario & $x$ & $x$ & $x$ & - & $45 \%$ & - & - & DA \\
\hline 17 & $M$ & 56 & Hospitalario & $x$ & $x$ & - & - & $50 \%$ & $x$ & NA & $C D$ \\
\hline 18 & $M$ & 47 & Hospitalario & $x$ & $x$ & - & - & $30 \%$ & $x$ & NA & OM1 \\
\hline 19 & M & 51 & Ambulatorio & $x$ & $x$ & - & - & $60 \%$ & NA & $x$ & DA \\
\hline 20 & $M$ & 57 & Hospitalario & $x$ & $X$ & $X$ & - & $60 \%$ & $x$ & NA & $C X$ \\
\hline 21 & $\mathrm{~F}$ & 77 & Ambulatorio & $x$ & $x$ & $x$ & - & $50 \%$ & NA & $x$ & $C X$ \\
\hline 22 & $F$ & 62 & Hospitalario & $x$ & $x$ & $x$ & - & $50 \%$ & $x$ & NO & DA-2 CD \\
\hline 23 & $\mathrm{~F}$ & 64 & Ambulatorio & - & $x$ & $x$ & - & - & NA & $x$ & $C D$ \\
\hline 24 & $M$ & 55 & Ambulatorio & $x$ & $x$ & $x$ & $x$ & $30 \%$ & NA & $x$ & $C D$ \\
\hline 25 & $\mathrm{~F}$ & 65 & Ambulatorio & $x$ & $x$ & - & - & - & $x$ & NA & $C D$ \\
\hline 26 & $M$ & 61 & Ambulatorio & $x$ & $x$ & - & - & - & $x$ & NA & $C D$ \\
\hline 27 & $\mathrm{~F}$ & 78 & Ambulatorio & $x$ & $x$ & $x$ & - & - & NA & $x$ & $C X$ \\
\hline 28 & M & 56 & Hospitalario & $x$ & $x$ & $\mathrm{X}$ & $x$ & $35 \%$ & $x$ & NA & DA \\
\hline 29 & M & 61 & Hospitalario & $x$ & $x$ & - & - & $45 \%$ & - & - & $C D$ \\
\hline 30 & $M$ & 56 & Ambulatorio & $x$ & $x$ & $x$ & - & $60 \%$ & - & - & $C X$ \\
\hline 31 & $\mathrm{~F}$ & 74 & Hospitalario & $x$ & $x$ & $x$ & $x$ & $35 \%$ & - & - & DA \\
\hline 32 & $\mathrm{~F}$ & 68 & Hospitalario & $x$ & $x$ & - & - & $50 \%$ & $x$ & NA & DA \\
\hline 33 & M & 75 & Ambulatorio & $x$ & $x$ & $x$ & $x$ & $30 \%$ & - & - & $C X$ \\
\hline 34 & $\mathrm{~F}$ & 68 & Hospitalario & $x$ & $x$ & $x$ & - & $30 \%$ & $x$ & NA & $C D$ \\
\hline 35 & M & 57 & Hospitalario & $x$ & $x$ & - & - & $35 \%$ & NA & $x$ & $C D$ \\
\hline 36 & $M$ & 59 & Ambulatorio & $x$ & $x$ & - & $x$ & $56 \%$ & $x$ & NA & DA \\
\hline
\end{tabular}

BMS: bare metal stent, stent metálico o stent convencional; CD: arteria coronaria derecha; CX: arteria circunfleja; DA: arteria descendente anterior; DES: drug eluting stent, stent liberador de droga o stent medicado; DM2: diabetes mellitus tipo 2; DP: arteria descendente posterior; Enf. cor.: enfermedad coronaria; ERC: enfermedad renal crónica; F: femenino; FEVI: fracción de eyección del ventrículo izquierdo; HTA: hipertensión arterial; M: masculino; OM1: arteria obtusa marginal 1; PL: arteria posterolateral; RI: arteria ramus intermedio; Susp. fco: suspensión de fármaco, indicando la relación principalmente con la suspensión de clopidogrel.

La edad está reportada en años.

descendente anterior $(43,5 \% ; n=17)$, coronaria derecha $(38,4 \% ; n=15)$, circunfleja $(12,8 \% ; n=5)$ y obtusa marginal y ramus intermedio $(2,5 \% ; n=1)$ para cada arteria (tabla 9$)$.

Dado el hallazgo incidental de la estenosis de los stent durante la revisión de las historias clínicas, se determinó mediante la utilización de EpiOpen v. 3 el análisis estadístico en tablas de $2 \times 2$ para obtener el resultado, y mediante $\chi^{2}$ la razón de disparidad entre los pacientes de trombosis y estenosis del stent como método para determinar la medición de posibilidad de trombosis.

Dentro del análisis estadístico, la hipertensión arterial fue la comorbilidad que más se halló en pacientes con estenosis del stent, a diferencia de los pacientes con trombosis del mismo: OR, 0,06 (IC 95\%: 0,006-0,73); $p=0,03$.
La enfermedad coronaria no permitió un adecuado cálculo de la razón de disparidad ni de la $p$, ya que todos los pacientes portadores de stent padecían, obviamente, enfermedad coronaria.

Otras condiciones que favorecieron la aparición de trombosis del stent, a diferencia de la estenosis del mismo, fueron el sexo masculino y el stent convencional: OR, 2 (IC 95\%: 0,36-10,92; $p=0,67$ ) y OR, 1,8 (IC 95\%: 0,41-7,75; $p=0,67$ ), respectivamente (tabla 10 ).

\section{Resultados finales de las fases I y ॥}

El objetivo principal de este trabajo fue establecer la frecuencia de trombosis del stent coronario en el Hospital 
Tabla 10 Medición de posibilidad de trombosis vs. estenosis del stent

\begin{tabular}{|c|c|c|c|c|c|}
\hline Análisis & $\mathrm{n}$ & Trombosis & Estenosis & OR (IC 95\%) & $\mathrm{p}$ \\
\hline \multicolumn{6}{|l|}{ Sexo } \\
\hline Masculino & 32 & 8 & 24 & $2(0,36-10,92)$ & 0,67 \\
\hline Femenino & 14 & 2 & 12 & $0,5(0,09-2,73)$ & 0,67 \\
\hline \multicolumn{6}{|l|}{ Comorbilidad } \\
\hline Hipertensión arterial & 42 & 7 & 35 & $0,06(0,006-0,73)$ & 0,03 \\
\hline Diabetes mellitus & 22 & 3 & 19 & $0,38(0,08-1,72)$ & 0,35 \\
\hline Enfermedad renal crónica & 12 & 1 & 11 & $0,25(0,02-0,24)$ & 0,36 \\
\hline \multicolumn{6}{|l|}{ Tipo de stent } \\
\hline Convencional & 15 & 5 & 10 & $1,8(0,41-7,75)$ & 0,67 \\
\hline Medicado & 23 & 5 & 18 & $0,55(0,12-2,39)$ & 0,67 \\
\hline
\end{tabular}

Universitario Clínica San Rafael. El trabajo se realizó en dos fases; la primera entre el 1 de enero de 2011 al 30 de noviembre de 2011 y la segunda entre el 1 de diciembre de 2011 al 31 de diciembre de 2012, abarcando un total de 24 meses. Se encontraron en total 20 pacientes con diagnóstico de trombosis del stent, dato que corresponde a una frecuencia del 2,7\%. Las comorbilidades más frecuentes fueron: enfermedad coronaria en el $100 \%$ de los pacientes, seguido por hipertensión arterial en el $65 \%$, diabetes mellitus en el $25 \%$ y enfermedad renal crónica en el 10\%. La suspensión de la medicación, en particular del clopidogrel, fue descrita como causa de la trombosis del stent únicamente en el $15 \%$ de los casos (tabla 11).

\section{Discusión}

La enfermedad coronaria sigue teniendo una incidencia anual elevada. Registros del Ministerio de la Protección Social en Colombia en 2007 la consideran la primera causa de muerte en hombres y mujeres mayores de 45 años, superando las muertes violentas y el cáncer, con una tasa de muerte de 107,3 por 100.000 habitantes, con cifras mundiales similares hasta del $22 \%^{1}$. Por esta razón, con el paso del tiempo han surgido avances médicos para tratar de disminuir estas cifras. Una pieza fundamental fue el inicio del intervencionismo coronario y la angioplastia con stent, los cuales generaron un impacto positivo en la reducción de las complicaciones y la mortalidad, pero que además introdujeron riesgos como la trombosis del stent, siendo el episodio agudo el que condiciona un pronóstico negativo a corto plazo, con una alta morbimortalidad.

Al respecto, se han implementado medidas como los stents liberadores de fármacos, reduciendo el $5 \%$ de los casos por intervenir ${ }^{8}$. Así mismo, se ha implementado el uso de antiagregación dual con antiplaquetarios, a sabiendas de que la variabilidad de los mismos entre distintos individuos es considerable y que, por tanto, el riesgo de trombosis aún sigue latente? .

Tabla 11 Características demográficas de los pacientes con diagnóstico de trombosis del stent en un periodo de dos años

\begin{tabular}{|c|c|c|c|c|c|}
\hline & Fase 1 & Fase 2 & Total & Promedio & $\%$ \\
\hline Total de angioplastias & 642 & 248 & 890 & 445 & - \\
\hline Total hombres & 400 & 185 & 585 & 292,5 & 65,70 \\
\hline Total mujeres & 242 & 63 & 305 & 153 & 34,50 \\
\hline Promedio total edad hombres angioplastias & 67,5 & 64,5 & - & 66 & - \\
\hline Promedio total edad mujeres angioplastias & 70,7 & 69,8 & - & 70,2 & - \\
\hline Trombosis del stent $(n)$ & 10 & 10 & 20 & 10 & - \\
\hline Frecuencia trombosis & $1,50 \%$ & $4 \%$ & - & $2,70 \%$ & - \\
\hline Total hombres trombosis & 6 & 8 & 14 & 7 & 70 \\
\hline Total mujeres trombosis & 4 & 2 & 6 & 3 & 30 \\
\hline Promedio edad hombres trombosis del stent & 63,3 & 62,8 & - & 63 & - \\
\hline Promedio edad mujeres trombosis del stent & 73,7 & 67 & - & 70,3 & - \\
\hline Hipertensión arterial trombosis stent & 6 & 7 & 13 & 6,5 & 65 \\
\hline Enfermedad coronaria trombosis stent & 10 & 10 & 20 & 10 & 100 \\
\hline Diabetes mellitus trombosis stent & 2 & 3 & 5 & 2,5 & 25 \\
\hline Enfermedad renal crónica trombosis stent & 1 & 1 & 2 & 1 & 10 \\
\hline Promedio FEVI-trombosis del stent & $36,80 \%$ & 40,7 & - & $38,70 \%$ & - \\
\hline Stent convencional trombosado & 4 & 6 & 10 & 5 & 45,50 \\
\hline Stent medicado trombosado & 6 & 6 & 12 & & 54,50 \\
\hline Suspensión de medicación trombosis del stent & 2 & 1 & 3 & 1,5 & 15 \\
\hline
\end{tabular}


En la revisión de la literatura se encontró una incidencia baja, que varió entre el $1,5 \%$ hasta el $2 \%$ en una revisión de $\operatorname{casos}^{8}$. En una fase inicial del estudio se describieron 642 procedimientos realizados en 11 meses, de los que el $1,6 \%$ (10 casos) desarrolló trombosis de los stents implantados. En la segunda fase durante 13 meses de seguimiento, se realizaron 1.458 procedimientos en el servicio de hemodinamia, de los cuales 1.230 correspondieron a enfermedad coronaria. De estos, 248 correspondieron a angioplastias con stent y se reportaron 10 casos de trombosis del stent, cifra que corresponde a una frecuencia del $4 \%$.

De otra parte, se hallaron asociaciones frecuentes, como que el $100 \%$ de los pacientes estaba hospitalizado debido a que consultó primariamente por urgencias, considerándose que el hallazgo de trombosis del stent es un factor de alto riesgo y mal pronóstico en la evolución de aquellos con enfermedad coronaria, en comparación con aquellos que acuden a un procedimiento electivo de forma ambulatoria, en quienes no se encontraron casos de trombosis del stent.

Así mismo, se halló que todos los casos de trombosis del stent cursaron con evento coronario agudo y evidencia de trastornos en la función ventricular izquierda, además de fracción de eyección promedio del ventrículo izquierdo del 40,7\%. De igual manera, el $80 \%$ correspondió al género masculino, con un promedio de edad de 63,7 años.

En un paciente se documentó la suspensión del consumo de clopidogrel, mientras que en el estudio anterior 2 pacientes lo habían hecho, lo que corresponde al $15 \%$ de los casos con trombosis del stent. En vista de que el diseño metodológico y la falta de información en las historias clínicas no permiten realizar una asociación directa, deben implementarse estudios posteriores prospectivos con el fin de determinar con exactitud este dato, pues por publicaciones previas se conoce que entre el 16 y el $50 \%$ suspendieron el tratamiento en el mes previo a la trombosis.

Como se mencionó, este estudio se desarrolló en dos fases, en un tiempo de 24 meses, en el cual se mantuvieron las mismas características demográficas para un total de 890 angioplastias más stent, de los cuales el 65,7\% correspondió al género masculino, encontrándose un total de 20 diagnósticos de trombosis, que corresponden a una frecuencia del $2,7 \%$. Se identificaron comorbilidades asociadas a enfermedad coronaria (100\%), hipertensión arterial (65\%), diabetes mellitus (25\%) y enfermedad renal (10\%). Estos datos se asemejan a los documentados en la población mundial, caracterizando la población colombiana del Hospital Universitario Clínica San Rafael.

Cabe resaltar que en la primera fase se determinó que la mayoría de los stents medicados se trombosan con una frecuencia del $60 \%$, a diferencia de lo encontrado en la segunda fase, en la que no se encontró diferencia significativa en cuanto se trataba de stent convencional o medicado. Por tanto, se considera conveniente realizar estudios adicionales en dicha población con el objetivo de establecer este como un factor de riesgo.

Durante la revisión de las historias clínicas se documentó un dato adicional susceptible de estudio, como fue la reestenosis de los stents, tema que si bien no constituyó el objetivo principal del estudio, fue significativo desde el punto de vista estadístico dada una frecuencia del $14,5 \%$ en el número total de angioplastias, de las cuales el 33,3\% correspondió a pacientes de género femenino, con un promedio de edad
$66,6 \%$, compartiendo en orden de continuidad las mismas comorbilidades vistas en los pacientes con trombosis. Aun así, se desconocen datos previos de la población colombiana, de ahí que se cree podría tratarse de un primer pilar para iniciar estudios adicionales.

\section{Conclusiones}

La frecuencia de trombosis del stent coronario en la población analizada es similar a la reportada en la literatura mundial. Este tipo de evento ocurre con mayor frecuencia en hombres, a una edad de aparición más temprana, aunada a un deterioro marcado de la función ventricular izquierda, en donde la hipertensión arterial y la diabetes mellitus constituyen las comorbilidades más asociadas.

Dado el tipo de estudio realizado (retrospectivo), se identificó como limitante la ausencia de seguimiento de los pacientes, lo que dificulta el cálculo de incidencia del evento, ya que aquellos con diagnóstico de trombosis del stent pudieron haber tenido el implante del mismo en otra institución y haber consultado en el hospital del estudio, o caso contrario, a quienes se les implantó el stent en el centro de estudio pudieron haber consultado por trombosis en otra institución.

Como hallazgo incidental se reportó una alta frecuencia de evento de reestenosis del stent, la cual requiere más estudios a futuro debido a la importancia e impacto que puede representar en cuanto a la necesidad de nuevas intervenciones y pronóstico de la enfermedad.

\section{Responsabilidades éticas}

Protección de personas y animales. Los autores declaran que para esta investigación no se han realizado experimentos en seres humanos ni en animales.

Confidencialidad de los datos. Los autores declaran que en este artículo no aparecen datos de pacientes.

Derecho a la privacidad y consentimiento informado. Los autores declaran que en este artículo no aparecen datos de pacientes.

\section{Financiación}

Ninguna.

\section{Conflicto de intereses}

Los autores declaran no tener conflictos de intereses.

\section{Agradecimientos}

Al Hospital Universitario Clínica San Rafael por permitirnos adelantar la investigación planteada y por ser pilar fundamental en nuestra formación como Especialistas en Medicina Interna.

A los Doctores Fernando Suárez, Wilson Suárez, Miguel Ángel Zuleta y Jorge Villegas por su constante apoyo, asesoría clínica y estadística para la realización de este trabajo. 


\section{Bibliografía}

1. Popma JJ, Bhatt DL. Braunwald's Heart Disease - A Textbook of Cardiovascular Medicine. 9th ed Philadelphia: Elselvier; 2012.

2. Baim D. What does the future hold? En: Symposium The Great debate on coronary drueluting stents. Toulose; 2007.

3. Sousa AG, Mattos LA, Campos Neto CM, Carvalho HG, Stella FP, Nunes $G$. Percutaneous myocardial revascularization procedures in Brazil in 1996-1997 compared to the 1992-1993 period: A report of the National Registry - National Center for Cardiovascular Interventions (CENIC). Arq Bras Cardiol. 1998;70:423-30.

4. Stone GW, Moses JW, Ellis SG, Schofer J, Dawkins KD, Morice $M C$, et al. Safety and efficacy of sirolimus- and paclitaxel-eluting coronary stents. N Engl J Med. 2007;356:998.

5. McFadden EP, Stabile E, Regar E, Cheneau E, Ong AT, Kinnaird T, et al. Late thrombosis in drug-eluting coronary stents after discontinuation of antiplatelet therapy. Lancet. 2004;364:1519-21.
6. Beltrán JR, Beltrán R, Caicedo VM, García M, García E, Gómez EA, et al. Guías colombianas de Cardiología. Síndrome coronario agudo sin elevación del ST. Rev Col Cardiol. 2008;15 Supl 3:143-232.

7. Morice MC, Serruys PW, Sousa JE, Fajadet J, Ban HE, Perin M, et al. A randomized comparison of a sirolimus-eluting stent with a standard stent for coronary revascularization. N Engl J Med. 2002; 346:1773-80.

8. Moses JW, Leon MB, Popma JJ, Fitzgerald PJ, Holmes DR, O’Shaughnessy C, et al. Sirolimus-eluting stents versus standard stents in patients with stenosis in a native coronary artery. $\mathrm{N}$ Engl J Med. 2003;349:1315-23.

9. Joner M, Finn AV, Farb A, Mont EK, Kolodgie FD, Ladich E, et al. Pathology of drug-eluting stents in humans: delayed healing and late thrombotic risk. J Am Coll Cardiol. 2006;48: 193-202. 